\title{
A serpentine laminating micromixer combining splitting/recombination and advection
}

\author{
Dong Sung Kim, ${ }^{a b}$ Se Hwan Lee, ${ }^{c}$ Tai Hun Kwon*ab and Chong H. Ahn ${ }^{c}$ \\ Received 6th December 2004, Accepted 24th March 2005 \\ First published as an Advance Article on the web 26th April 2005 \\ DOI: 10.1039/b418314b
}

Mixing enhancement has drawn great attention from designers of micromixers, since the flow in a microchannel is usually characterized by a low Reynolds number $(R e)$ which makes the mixing quite a difficult task to accomplish. In this paper, a novel integrated efficient micromixer named serpentine laminating micromixer (SLM) has been designed, simulated, fabricated and fully characterized. In the SLM, a high level of efficient mixing can be achieved by combining two general chaotic mixing mechanisms: splitting/recombination and chaotic advection. The splitting and recombination (in other terms, lamination) mechanism is obtained by the successive arrangement of " $F$ "-shape mixing units in two layers. The advection is induced by the overall three-dimensional serpentine path of the microchannel. The SLM was realized by SU-8 photolithography, nickel electroplating, injection molding and thermal bonding. Mixing performance of the SLM was fully characterized numerically and experimentally. The numerical mixing simulations show that the advection acts favorably to realize the ideal vertical lamination of fluid flow. The mixing experiments based on an average mixing color intensity change of phenolphthalein show a high level of mixing performance was obtained with the SLM. Numerical and experimental results confirm that efficient mixing is successfully achieved from the SLM over the wide range of $R e$. Due to the simple and mass producible geometry of the efficient micromixer, SLM proposed in this study, the SLM can be easily applied to integrated microfluidic systems, such as micro-total-analysis-systems or lab-on-a-chip systems.

\section{Introduction}

Recently, a wide range of applications of microfluidic systems is currently being witnessed in the fields of miniaturized analytical systems for chemistry and biology, such as genomic and proteomic analysis, clinical diagnostics and micro-totalanalysis-systems. ${ }^{1-3}$ Mixing plays a very important role in these microfluidic analysis systems. ${ }^{2,3}$ For fast diagnosis results, efficient mixing of reagents is definitely desired. Because of the low Reynolds number $(R e)$ of the microchannel flow in miniaturized analytical systems, it is impossible to expect turbulent mixing of two fluids. If mixing is obtained only by a diffusion mechanism, total mixing time could be estimated by rearranging Fick's law as follows:

$$
t \sim d^{2} / D
$$

where $t, d$ and $D$ are the time needed to obtain whole mixing, the thickness of the lamellar structure, and the diffusion coefficient, respectively. If diffusion is the only mixing mechanism, the mixing time becomes equivalent to several minutes for the case of a lamellar structure thickness of the order of $100 \mu \mathrm{m}$ and $D$ of the order of $10^{-10} \mathrm{~m}^{2} \mathrm{~s}^{-1}$. In this regard, numerous mixing schemes to obtain efficient mixing performance in the microchannel were proposed: e.g. Coanda effect by means of the arrangement of modified Tesla

*thkwon@postech.edu structures, ${ }^{4}$ transverse flow of material elements caused by slanted well, ${ }^{5}$ and various chaotic mixing mechanisms. ${ }^{6-14}$

Chaotic mixing in a laminar flow regime can remarkably enhance the mixing efficiency with a relatively small pressure increase. Time periodic perturbation imposed on two-dimensional flow, which is a kind of active mixing, results in chaotic flow in the microchannel. For instance, Lee et al. ${ }^{6}$ obtained chaotic mixing by adding a periodic pressure perturbation to two-dimensional laminar flow. In this case, however, an extra energy source is required to cause periodic perturbation to the main stream. In contrast, just a spatially periodic perturbation in three-dimensional flow can also cause chaotic flow in a passive manner. Geometrically periodic perturbation induces stretching and folding of material elements, consequently resulting in chaotic mixing in fluid flows. ${ }^{15-17}$ Liu et al. ${ }^{7}$ achieved chaotic mixing by a three-dimensional serpentine microchannel whose design causes chaotic advection. In this case, however, relatively high $R e$ is needed to get the effect of chaotic advection due to the inertia effect dependency of advection. Stroock et al. ${ }^{8}$ showed that oblique ridges can cause three-dimensional helical flow in the microchannel. In addition, they obtained chaotic mixing by alternating velocity fields induced by staggered herringbone structures on the bottom wall of the microchannel. Kim et al. ${ }^{9}$ presented a chaotic micromixer, in which chaotic mixing is achieved by periodic perturbation of a helical type of flow obtained by slanted grooves. Periodically inserted barriers along the channel wall in the micromixer impose alternating velocity fields with periodic existence of a hyperbolic point, consequently resulting 
in a chaotic mixing. Bertsch et al. ${ }^{10}$ realized chaotic mixing via a micro-stereolithography technology by miniaturizing conventional Kenics $(\mathrm{IM})$ and SMX $\mathrm{IM}$ mixers in which the chaotic mixing mechanism is based on the splitting and reorientation of fluid flows. Kim et al. ${ }^{11}$ also suggested a chaotic micromixer that combines two chaotic mixing mechanisms: the splitting/ reorientation of the conventional Kenics mixer and stretching/ folding via periodically located barriers.

On the other hand, splitting and recombination (in other terms, lamination) of flow, as one of the chaotic mixing mechanisms, could remarkably enhance the mixing efficiency by increasing interfacial area exponentially over a wide range of $R e .^{12-14}$ Branebjerg et al. ${ }^{12}$ successfully obtained multilayer lamination by introducing a "separation plate" inside the microchannel. They realized the micromixer in a bonded silicon/glass sandwich in which the separation plate was achieved via an etchstop layer. At a similar time, Schwesinger et al. ${ }^{13}$ obtained relatively good mixing without the separation wall, via arranged fork-shape mixing elements fabricated by anisotropic bulk wet etching of two silicon wafers. However, due to the opaqueness of the silicon wafers, they couldn't observe the mixing behaviors inside their micromixer. Recently, Schönfeld et al. ${ }^{14}$ showed that, in order to get the ideal lamination, a splitting intermediate layer (separation wall) inside the channel should be needed. They optimized their mixer by inserting a splitting layer of a lasermachined stainless steel sheet inside the microchannel which was fabricated by the milling process. According to their study, the separation wall might be required to realize the ideal lamination. However, it is hardly achievable to realize the mass production of micromixers with the separation wall inside the microchannel using polymers via, e.g., injection molding in view of the producibility and mechanical strength of the thin separation wall. For chemical processing applications such as microreactors, polymer materials could not be applied, especially for applications including solvents or gas phase reactions. For specific chemical processing applications, such as miniaturized chemical production plants, costly microsystems via small-series fabrication are still competitive enough. ${ }^{3}$

However, for diagnostic devices dealing with blood, for instance, disposability is usually required, and therefore low cost devices via mass production become more competitive. In view of mass production, a simple design is better than a complex one as long as comparable performance could be achieved via the simple design. In this regard, it is of great interest to improve the lamination of the fluid flow inside the microchannel to remarkably enhance the mixing performance without a separation wall, thereby realizing the micromixer via mass production methods. With this in mind, in the following sections, we propose a newly designed micromixer that favorably combines the two general chaotic mixing mechanisms of "splitting and recombination" by the successive arrangement of "F"-shape mixing units in two layers and "advection" by means of the overall three-dimensional serpentine channel path. In addition, the simple geometry of the proposed micromixer would reduce undesirable entrapment or nonspecific surface adsorption ${ }^{18}$ of cells or antibodies (or micro/nano sized particles in some cases) on the channel surface, which is a critical issue in applications involving antigen-antibody reactions. To confirm the proposed mixing mechanism, a numerical mixing simulation is conducted. Mixing experiments by means of a $\mathrm{pH}$ indicator are also carried out. Finally, numerical and experimental results are presented showing the higher level of efficient mixing via the improved lamination in the proposed micromixer.

\section{Design and simulation}

\section{Design of micromixer}

Fig. 1(a) shows a schematic diagram of the proposed efficient micromixer in this study. The micromixer is composed of successively arranged " $F$ "'-shape mixing units in two layers. Furthermore, the overall microchannel path of the arranged mixing units forms a three-dimensional serpentine path. The successive arrangement of F-shape mixing units causes continuous splitting and recombination, i.e. lamination of the fluid flow, keeping the same flow path length for the two splitting streams. Therefore, it may result in an exponential interfacial area growth as depicted in Fig. 1(b) of which the indicated positions correspond to cross-sections in Fig. 1(a). However, the actual splitting and recombination behaviors inside the micromixer could not be the same as in Fig. 1(b) due to the absence of the separation wall. The three-dimensional serpentine design of the micromixer also induces a stirring effect at the corners due to the advection as explained above. Interestingly, the advection of the serpentine design acts positively to realize the ideal lamination of flow which could not otherwise be realized without the separation wall (discussed in more detail later). Therefore, we have favorably combined two efficient mixing mechanisms in the proposed micromixer which could remarkably enhance the mixing

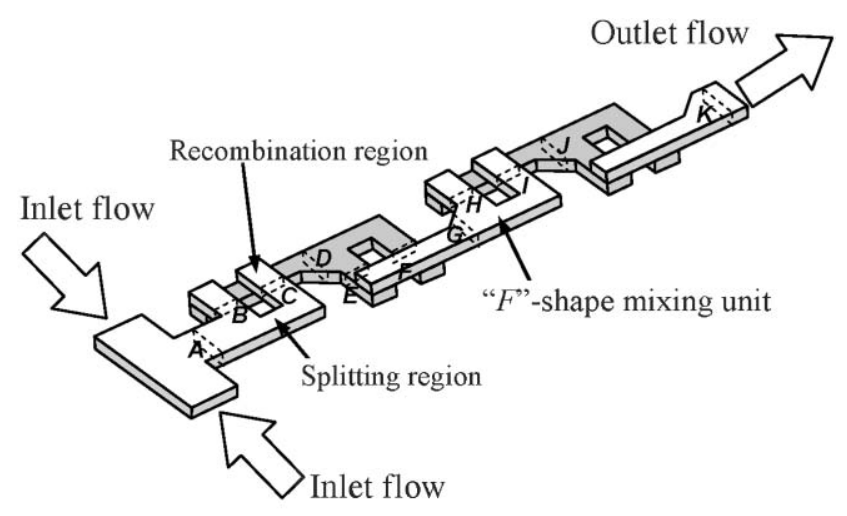

(a)

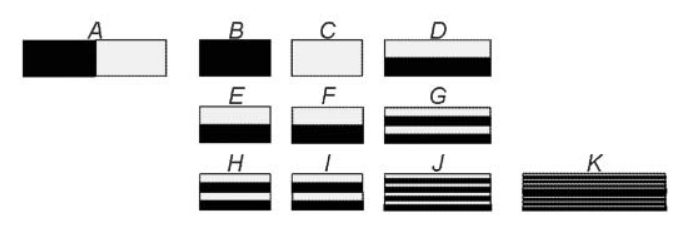

(b)

Fig. 1 Serpentine laminating micromixer (SLM): (a) schematic diagram of the SLM and (b) the conceptual cross-sectional mixing behaviors of the ideal vertical lamination at each indicated position. 
performance inside the microchannel over a wide range of $R e$. In this paper, we named the proposed efficient micromixer a serpentine laminating micromixer (SLM).

It is worthwhile to note that the SLM can be easily realized by bonding of two mass-produced layers, made e.g. via injection molding, containing upper and lower F-shape mixing units, because it does not contain an intermediate thin separation layer inside the channel. Furthermore, the vertical lamination method of the SLM is the most promising method of lamination mixing for a high width-to-thickness rectangular cross-sectional channel, as usually observed in microchannels fabricated by conventional photolithography, since it can generate a larger interfacial area in width (in other words, it can remarkably reduce the thickness of lamella structures to be mixed), in contrast to the triangular cross-sectional channel of the work of Schwesinger et al. ${ }^{13}$ It might be mentioned that the arranged fork-shape mixing elements of Schwesinger et al.'s work $^{13}$ may give rise to unmixed dead volume due to the different flow path lengths of the two splitting streams.

\section{Numerical mixing simulation}

In the SLM, the two efficient mixing mechanisms of splitting/ recombination and advection are favorably combined to realize better lamination of the fluid flow without a separation wall. In order to evaluate the mixing behavior inside the SLM, computational fluid dynamics (CFD) mixing simulations under the steady-state condition were conducted based on the finite volume method by means of CFD-ACE+ (CFD Research Corporation, AL, USA). In the CFD simulations, a total of four arranged F-shape mixing units (i.e., four splitting and recombination regions) with two inlets and one outlet of the flow, as schematically shown in Fig. 1(a), were chosen as a computational domain. The width and height of the main channel were $250 \mu \mathrm{m}$ and $60 \mu \mathrm{m}$, respectively, and those of the inlets and outlet were $500 \mu \mathrm{m}$ and $60 \mu \mathrm{m}$, respectively. The total length of the SLM was $6.5 \mathrm{~mm}$ in the computational domain. The computational domain was divided into discrete elements by three-dimensional structured grids and the total numbers of nodes and volume elements were 68749 and 57056, respectively. Furthermore, to numerically compare the mixing performance of the SLM with another micromixer, a T-type micromixer which has the same width, height and length as the SLM was also designed and divided into discrete elements by three-dimensional structured grids. The total numbers of nodes and volume elements of the T-type micromixer were 29736 and 24888, respectively.

The physical properties of water were chosen as those of the working fluid. The diffusion coefficient of a solute (species) in water was set to $10^{-10} \mathrm{~m}^{2} \mathrm{~s}^{-1}$. The first order upwind scheme was used for spatial differencing of velocity and species. The conjugate gradient squared and preconditioning (CGS+Pre) solver was applied to velocity correction and species, and the algebraic multigrid (AMG) solver was used for pressure correction. Boundary conditions of constant normal flow velocities and fixed pressure were assigned at the inlets and outlet, respectively.

The mixing performance of the T-type micromixer and the SLM were compared at a flow rate of $50 \mu \mathrm{min}^{-1}(R e \approx 3.89)$. To investigate the advection effect on the lamination behavior of the flow according to the $R e$ inside the SLM, we extensively carried out the mixing simulations by changing the flow rate to 3.6, 18, 36, 180 and $360 \mu \mathrm{min}^{-1}$ (the corresponding Re are $0.28,1.40,2.80,14.0$ and 27.99 , respectively), and then we observed the cross-sectional lamination behavior at the recombination region. The detailed results will be discussed later.

\section{Experimental}

\section{Fabrication of micromixer}

As already mentioned above, to realize the SLM in mass production is one of the principal objectives of this work. The SLM was realized by means of an injection molding process, as one of the most promising mass production methods of polymer materials. Fig. 2 illustrates the schematic diagram of the fabrication process of the SLM. Two nickel disks were prepared as mold inserts for the injection molding process, of which the diameter and thickness were 3 inch and $1.5 \mathrm{~mm}$, respectively. The nickel disks were cleaned and then dried in a $120{ }^{\circ} \mathrm{C}$ oven for about $2 \mathrm{~h}$ before the spin coating process. In order to improve the adhesion between the nickel surface and the thick negative photoresist of SU-8, first, OmniCoat $\mathrm{TM}$ (Microchem Corp., MA, USA) was spin coated over the nickel disks at a speed of $3000 \mathrm{rpm}$ for $30 \mathrm{~s}$ and baked on the hot plate at $200{ }^{\circ} \mathrm{C}$ for $1 \mathrm{~min}$. Then, the SU-8 2075 (Microchem Corp., MA, USA) was spin coated on the baked thin OmniCoat $(\mathrm{MM}$ layer at a speed of $950 \mathrm{rpm}$ for $45 \mathrm{~s}$, and then a soft baking process was carefully performed: ramping from 25 to $65{ }^{\circ} \mathrm{C}$ for $40 \mathrm{~min}$, holding at $65{ }^{\circ} \mathrm{C}$ for $10 \mathrm{~min}$, ramping from 65 to $95^{\circ} \mathrm{C}$ for $30 \mathrm{~min}$, and finally holding at $95^{\circ} \mathrm{C}$ for $1 \mathrm{~h}$ and $45 \mathrm{~min}$. After UV exposure with a dose of $250 \mathrm{~mJ} \mathrm{~cm}{ }^{-2}$, a post exposure baking process was carried out at $65{ }^{\circ} \mathrm{C}$ for $10 \mathrm{~min}$ and at $95{ }^{\circ} \mathrm{C}$ for $10 \mathrm{~min}$. Then, both $\mathrm{SU}-8$ and
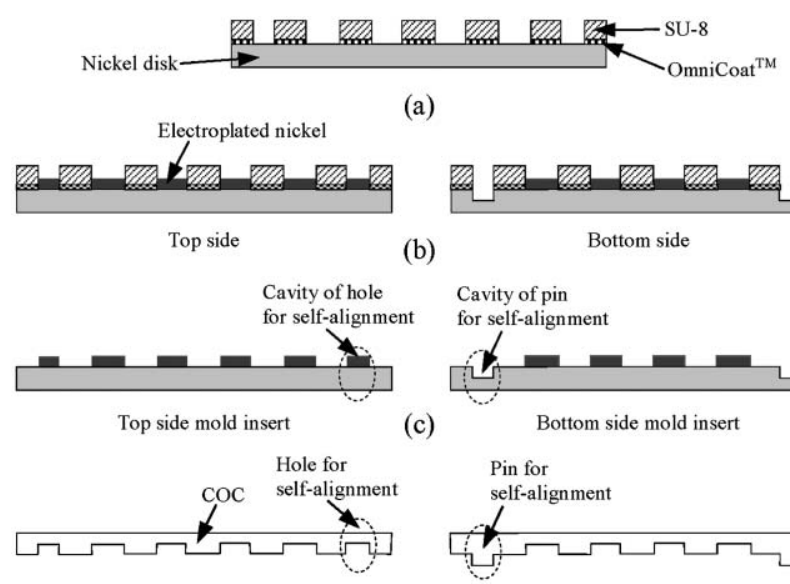

(c)

a)

(b)

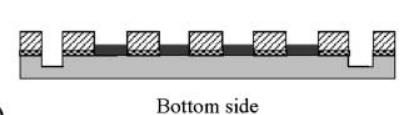

Cavity of pin

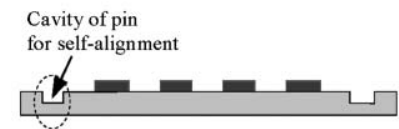

Bottom side mold insert

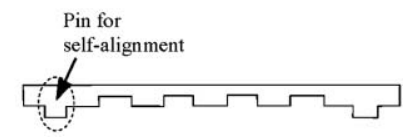

(d)

Bottom side COC substrate

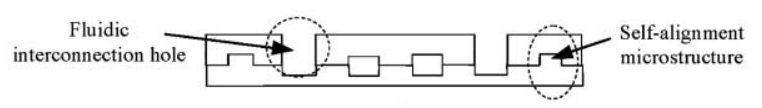

(e)

Fig. 2 Fabrication process of the SLM: (a) SU-8 and OmniCoat $(\mathrm{IM}$ layers patterned on nickel disk as masters for electroplating, (b) electroplated nickel in the masters, (c) nickel mold inserts after removing SU-8 and OmniCoat $\mathrm{PI}$ layers, (d) injection molded COC substrates and (e) SLM after thermal bonding. 
OmniCoat $(\mathrm{M} M$ were developed to obtain masters for the electroplating process (Fig. 2(a)). The electroplating process was then performed in a nickel electroplating bath at a current density of $10 \mathrm{~mA} \mathrm{~cm}^{-2}$ for $6 \mathrm{~h}$ at $60^{\circ} \mathrm{C}$ (Fig. 2(b)). Finally, two nickel mold inserts for the injection molding process, which contain $60 \mu \mathrm{m}$ thick electroplated nickel microstructures on a nickel disk, were obtained by stripping of the residual SU-8 photoresist and OmniCoat $(\mathrm{IM})$ as depicted in Fig. 2(c). It should be noted here that in order to realize the precise alignment of two polymer layers of the SLM during the bonding stage, cavities of pairs of pins and holes as the self-alignment microstructures were patterned on two nickel mold inserts as indicated in Fig. 2(c). ${ }^{19}$ One may refer to ref. 18 for the detailed process of this self-assembly technique.

The two polymer substrates of the SLM were replicated by injection molding of cyclic olefin copolymer (COC) by means of the two fabricated nickel mold inserts (Fig. 2(d)). It might be mentioned that the cycle time of the injection molding process was just about $1 \mathrm{~min}$ to replicate two COC substrates. Finally, the SLM was realized by the thermal bonding of the two injection molded COC substrates by means of a hot embossing machine after drilling holes for fluidic interconnection for inlets and outlet on the top COC substrate as shown in Fig. 2(e). During the bonding process, two replicated COC substrates were precisely aligned via the self-alignment microstructures as indicated in Fig. 2(e).

Fig. 3 shows an enlarged microscope view of the successive arrangement of F-shape mixing units in the fabricated injection molded COC SLM with detailed dimensions. A total of eight arranged F-shape mixing units were fabricated in the present SLM so that the length of the SLM became $10 \mathrm{~mm}$. The width and height of the main channel are $250 \mu \mathrm{m}$ and $60 \mu \mathrm{m}$, respectively, as in the simulation.

\section{Mixing experimental setup}

Fig. 4(a) and (b) show the experimental setup for characterizing mixing performance inside a micromixer and an enlarged view of the SLM equipped in the experimental setup, respectively. For the mixing experiment with pressure-driven flow, two flows containing different fluids were injected into the micromixer by a syringe pump (kdScientific Inc., KDS200) at a preset constant flow rate, as indicated in Fig. 4(a). In this mixing experiment, two solutions of $0.31 \mathrm{~mol} \mathrm{l}^{-1}$ phenolphthalein and $0.33 \mathrm{~mol}^{-1} \mathrm{NaOH}$ in $99 \%$ ethanol were

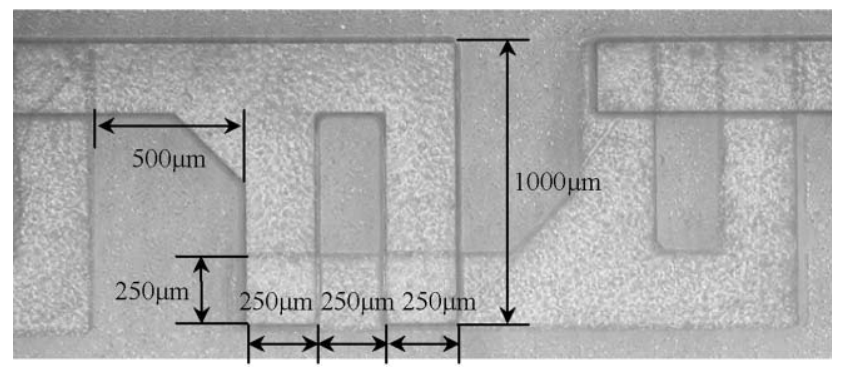

Fig. 3 Enlarged microscope view of successive arrangement of F-shape mixing units in two bonded COC (cyclic olefin copolymer) substrates with detailed dimensions (thickness of each F-shape mixing unit is $60 \mu \mathrm{m})$.

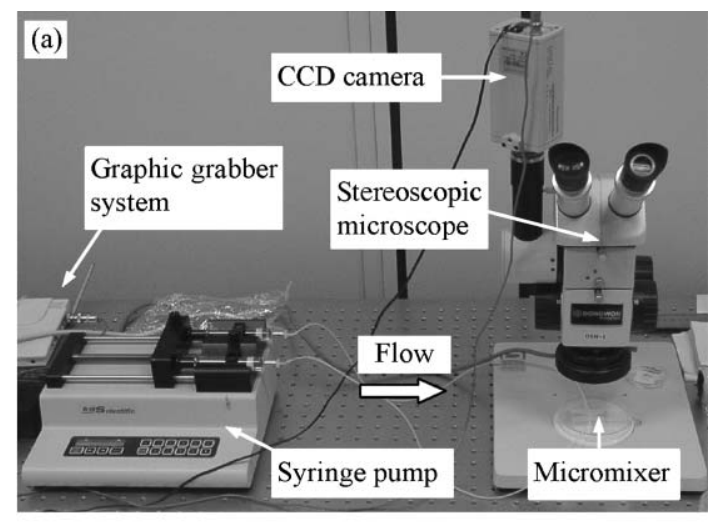

(b)

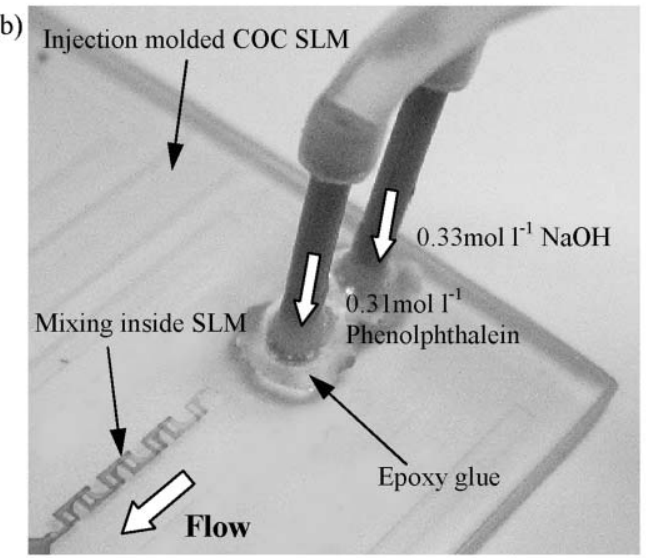

Fig. 4 Experimental setup for characterizing mixing performance inside a micromixer: (a) real overview and (b) enlarged view of the SLM equipped in the experimental setup. The red portion (dark grey in this figure) indicates the interface (mixed area) of the phenolphthalein and $\mathrm{NaOH}$ streams.

injected into the fabricated SLM through each inlet. ${ }^{7,9,11}$ $\mathrm{NaOH}$ solution showed a $\mathrm{pH}$ value of about 13 . Phenolphthalein is a $\mathrm{pH}$ indicator so that its color changes from colorless (transparent) to red if its $\mathrm{pH}$ becomes greater than 8 . Only the interface between the phenolphthalein and $\mathrm{NaOH}$ streams turns red, thereby enabling us to measure the mixing performance along the downstream as shown in Fig. 4(b).

In order to investigate the effect of the flow rate, i.e. Re, on the mixing performance, several experiments were carried out with various flow rates for the SLM. Nine different flow rates were selected: 10, 20, 50, 80, 100, 150, 200, 240 and $280 \mu 1 \mathrm{~min}^{-1}$ (the corresponding $R e$ are $0.44,0.88,2.19,3.51,4.39,6.58$, $8.77,10.53$ and 12.28 , respectively).

The mixing patterns in the SLM were observed with time and it was found that it took less than a second to reach the steady state flow from the time when the two streams enter the T-junction of the SLM. Once the steady state was reached, the color change along the downchannel direction was captured by means of a stereoscopic microscope (DONGWON EnC Corp., OSM-1) at ten times magnification with a CCD camera (Honeywell Inc., GC-405NA) and a graphic grabber system (Sasem Co. Ltd., OnAir TV2) installed on a personal computer. A white light lamp was placed right above the SLM as an illumination source. 


\section{Results and discussion}

\section{Numerical mixing simulation results}

Fig. 5 shows the numerical mixing simulation results of the T-type micromixer and the SLM at a flow rate of $50 \mu \mathrm{min}^{-1}$ $(\operatorname{Re} \approx 3.89$ ). The simulation results show the superiority of the SLM over the T-type micromixer as expected. In the T-type micromixer, the interface first formed at the $\mathrm{T}$-junction of the channel was maintained up to the exit of the channel as indicated in Fig. 5(a). Therefore, it was found that, with the T-type micromixer, it is hard to get a good mixing at the exit even with a channel length of $6 \mathrm{~mm}$ as shown in Fig. 5(a) for the given conditions, i.e., diffusivity, channel width and residence time (flow rate). In contrast, an almost complete mixing was achieved at the exit of the SLM with the same length as shown in Fig. 5(b). It is worthwhile to note that the first interface formed at the T-junction of the SLM flows into the first splitting channel as indicated in Fig. 5(b). To observe the interesting flow characteristic at the first splitting region, we have also carried out numerical simulations for the various Re. Fig. 6 shows the CFD simulation results according the change of the Re. As Fig. 5(b) shows, the first interface which was formed at the T-junction flows into the first splitting channel when $R e$ is small. In contrast, the first interface tends to flow into the second splitting channel at high $R e$ of 223.9 as

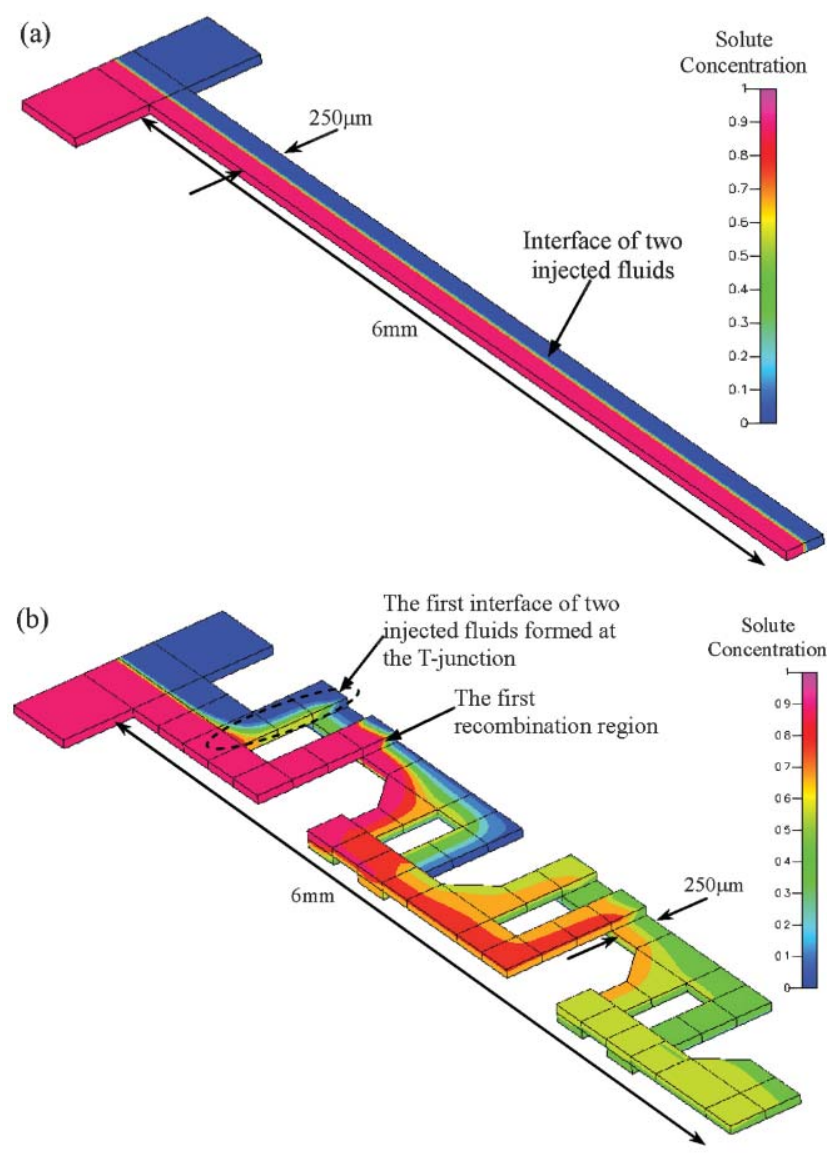

Fig. 5 Numerical mixing simulation results at the flow rate of $50 \mu \mathrm{lmin}^{-1}$ (the corresponding $R e \approx 3.89$ ): (a) T-type micromixer and (b) SLM.

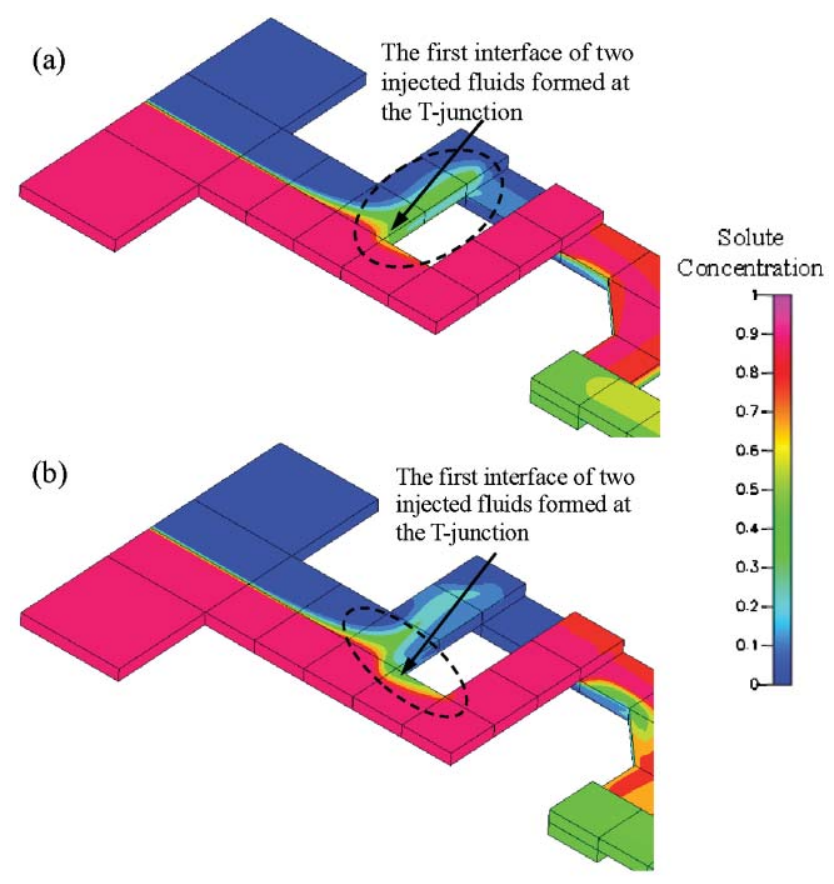

Fig. 6 CFD simulation results of flow patterns of the first interface at the first splitting region of the SLM for Re of: (a) 83.96 and (b) 223.9.

indicated in Fig. 6(b). In the case of intermediate range of $R e$ of 83.96, however, the motion of the interface is not so clear as indicated in Fig. 6(a).

It may be mentioned here that, however, due to the limitation in the fineness of the grid size in the present numerical models, the simulation results might have computational errors in computing mixing behavior due to numerical diffusion. ${ }^{20,21}$ In this regard, one may not get a quantitatively complete mixing in practical applications as in the simulation result (Fig. 5(b)) but one can qualitatively estimate the mixing performance inside the micromixer using the present models and simulations.

Fig. 7 shows the cross-sectional lamination behavior of fluid flow right after the first recombination region of the SLM for several $R e$ 's. For the case in Fig. 7(a), Re was maintained at 0.28 , and therefore it is expected that the mixing inside the SLM resulted only from the splitting and recombination effect

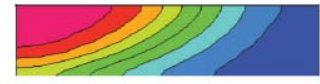

(a)

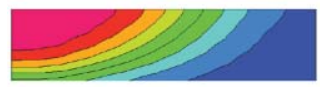

(c)

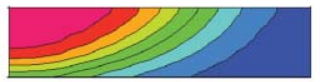

(b)

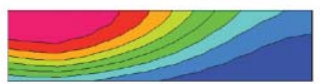

(d)

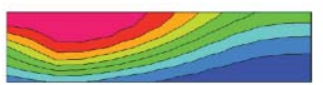
Solute
Concentration

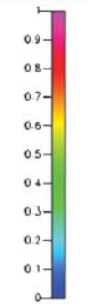

(e)

Fig. 7 Cross-sectional simulation results of mixing patterns of fluid flow right after the first recombination region of the SLM for Re of: (a) 0.28 , (b) 1.40 , (c) 2.80 , (d) 14.0 and (e) 27.99 . 
and not from the advection effect due to the negligible inertia force. Due to the absence of the separation wall, perfect vertical lamination could not be achieved at the low $R e$ shown in Fig. 7(a), as expected. However, as $R e$ increases, the inertia force effect is more involved and, interestingly, the crosssectional mixing behavior becomes more like the perfect vertical lamination with the help of the advection, as shown in Fig. 7(b)-(e). With a small advection effect, the interface is located diagonally rather than horizontally (Fig. 7(a)). In contrast, however, with a large advection effect the interface tends to become horizontal (Fig. 7(e)). In other words, the advection effect of the serpentine channel design acts favorably to realize the ideal lamination of flow as recognized in this study. It might be mentioned that since the detailed mixing behaviors cannot be observed after the second recombination region due to the numerical diffusion of the present model, as mentioned above, the mixing behaviors after only the first recombination region were presented. But one might expect a similar lamination effect to take place in the next recombination region due to the periodicity, i.e., successive arrangement of F-shape mixing units, of the present SLM design.

\section{Comparison of experimental mixing performance}

Fig. 8 shows experimental mixing results for the T-type micromixer and the SLM. We used experimental results for the T-type micromixer from Kim et al.'s work: ${ }^{9}$ the width, height and length of the T-type micromixer were $240 \mu \mathrm{m}, 60 \mu \mathrm{m}$ and $21 \mathrm{~mm}$, respectively; we used the first $10 \mathrm{~mm}$ of the channel to compare the result with that of the SLM. As mentioned before, when phenolphthalein meets and thereby reacts with $\mathrm{NaOH}$, it changes its color from colorless to red so that the interface between the two streams can be identified by the red color. In the case of the T-type micromixer, two streams flowing in parallel meet each other at the center of the channel and thus the interface is clearly observed from the T-junction of the channel as shown in Fig. 8(a). The thickness of the red portion gradually increases along the downchannel due to pure diffusion (Fig. 8(a)).

It should be noted that the intensity of red color indirectly indicates the level of mixing in the microchannel: good mixing will generate multiple layers of reacted phenolphthalein molecules across the thickness; when observed from the top of the channel, the intensity of red color increases with the number of layers of reacted phenolphthalein molecules due to the incoherent nature of white light that was used as the illumination source. ${ }^{7,9,11}$ In this regard, Fig. 8(b), showing the experimental results from the SLM, indicates that an almost complete mixing has been successfully achieved at the end of the channel. By naked eye, one can easily tell the superiority of the SLM over the T-type micromixer. It is interesting to note that the first interface formed at the T-junction flows into the first splitting channel, which is the same trend seen in the numerical mixing simulation, as indicated in Figs. 8(b) and 5 (b), respectively.

In order to compare between mixers and investigate the effect of $R e$ on mixing performance, a mixing measure would be required to quantify the degree of mixing. As such a measure, the red color intensity was adopted in this study. The red color intensity was measured along the downstream of the SLM. Eight graphic images right after eight recombination regions were captured and the background area outside the mixer was also captured for compensation purposes. The intensities of red color for all captured images were measured pixel by pixel, and then the measured intensity for each region was normalized as follows: $:^{9,11}$

$$
\bar{I}=\frac{\sum_{n=1}^{N} I_{n} / N-\bar{I}_{\mathrm{ref}}}{I_{\mathrm{max}}-\bar{I}_{\mathrm{ref}}}
$$

where $\bar{I}, I_{n}$ and $N$ represent the normalized average intensity, intensity at pixel $n$ and total number of pixels in the mixing zone of each captured image, respectively. $\bar{I}_{\text {ref }}$ represents the mean reference intensity for compensation purpose. $I_{\max }$ is the

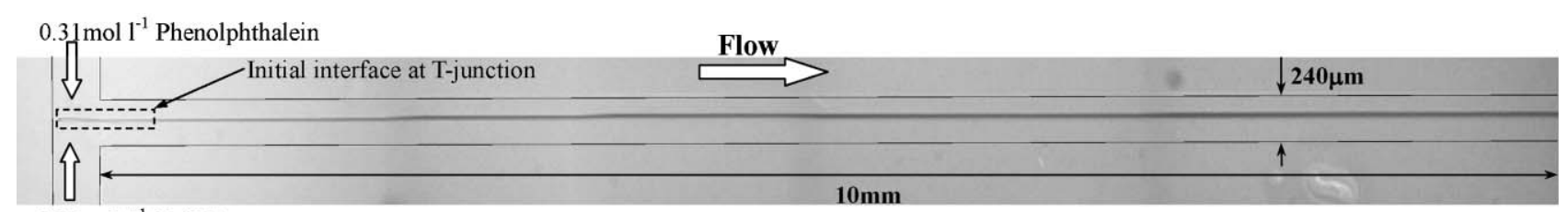

$0.33 \mathrm{~mol} \mathrm{l}^{-1} \mathrm{NaOH}$

(a)

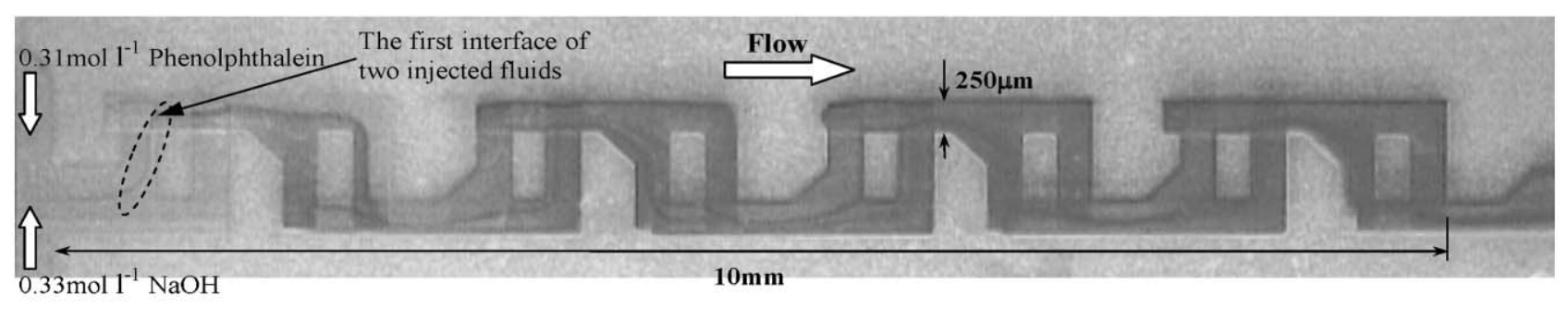

(b)

Fig. 8 Mixing experimental results of: (a) T-type micromixer $(R e \approx 2.28)$ from Kim et al.'s work ${ }^{9}$ and (b) SLM (Re $\left.\approx 2.19\right)$, at the same flow rate of $Q=50 \mu \mathrm{min}^{-1}$. Only the reacted phenolphthalein at the interface between the phenolphthalein and NaOH streams shows as a red color (dark grey in this figure). 
maximum intensity amongst pixels near the exit zone of the SLM in each experiment for normalization purposes. In eqn. (2), $\sum_{n=1}^{N} I_{n} / N$ means the average of intensity data in the mixing zone of each captured image and its change of value represents the change in degree of mixing along the downchannel direction. Even though the mixing experiments of the SLM were carried out in the same conditions, slight change of the illumination environment could cause overall intensity variation. The term $\bar{I}_{\text {ref }}$ in eqn. (2) was introduced to compensate for this intensity variation. The background transparent area (no mixing zone) outside the SLM in each experiment was used as a reference image to calculate the mean reference intensity value, which was calculated by $\bar{I}_{\text {ref }}=\sum_{n=1}^{N_{\text {ref }}} I_{\text {ref }, n} / N_{\text {ref }}$ (where $I_{\text {ref }, n}$ and $N_{\text {ref }}$ are intensity value at pixel $n$ and total number of pixels in the reference image, respectively). Therefore, according to eqn. (2), the normalized average intensity values of completely mixed and unmixed transparent zones become one and zero, respectively.

Plotted in Fig. 9 are the normalized average intensity changes, based on eqn. (2), along the downchannel direction for the SLM at several values of Re. To compare the mixing performance and show efficient mixing inside the SLM, we include the red color intensity variation data of the T-type micromixer at a flow rate of $50 \mu 1 \mathrm{~min}^{-1}(R e \approx 2.28)$ from Kim et al.'s work. ${ }^{9}$ Each calculated normalized intensity value was plotted at the abscissa corresponding to the center of each captured region. As expected, the average intensities of the SLM grow much faster than that of the T-type micromixer as shown in Fig. 9, indicating efficient mixing inside the SLM. The effective mixing mechanism of splitting and vertical recombination results in efficient mixing performance of the SLM even at low $R e$. It is worthwhile to mention that as $R e$ increases, the downchannel velocity increases and the residence time decreases. Due to this reduced residence time, the mixing

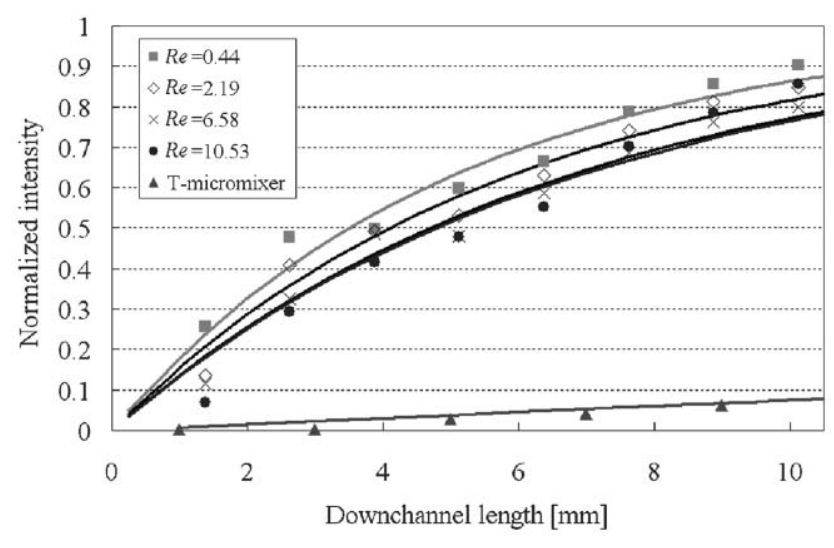

Fig. 9 Normalized average intensity changes along the downchannel direction of the SLM for $R e$ of $0.44,2.19,6.58$ and 10.53 (the corresponding flow rates are 10, 50, 150 and $240 \mu \mathrm{l} \mathrm{min}{ }^{-1}$, respectively) and the T-type micromixer for $R e$ of 2.28 (flow rate is $\left.50 \mu \mathrm{min}^{-1}\right)$. symbols: experimental data, and curves: optimal fitting with $\bar{I}=1-\exp (-z / \lambda)$. The optimal fitting parameters $\lambda$ (denoting the characteristic required mixing length, see below) of the SLM are 5.06, 5.90, 6.77 and $6.92 \mathrm{~mm}$, respectively, and that of the T-type micromixer is $129.7 \mathrm{~mm}$. intensity generally decreases as $R e$ increases. However, it should be noted that the intensity level near the exit of the SLM is high for the whole range of $R e$ in this study as shown in Fig. 9. It can be explained as follows: at higher $R e$, the advection induced by the serpentine channel path favorably affects the mixing to realize the ideal vertical lamination, as explained in Fig. 7; it results in more efficient lamination and reduces the thickness of lamellar structures to be mixed; the required mixing time is reduced and therefore, the mixing intensity can be maintained at high levels despite the decrease of residence time with the increase of $R e$. Therefore, the combined efficient mixing mechanisms of splitting/recombination and advection in the SLM enable us to maintain the high level of mixing performance for the whole range of $R e$ in this study even though the residence time decreases as $R e$ increases.

\section{Characteristic required mixing length}

For a quantitative comparison of mixing performance, a representative value that indicates the mixing performance is desired. It is well known that the stretching of material element or interfacial area increases exponentially with channel length due to chaotic mixing. ${ }^{15}$ It was of our interest to find the required mixing length from our own experimental data shown in Figs. 8 and 9. We defined a characteristic length scale as a length that appears as an exponent when the intensity data are fitted in terms of an exponential function. ${ }^{9,11}$ That is, the normalized average intensity, $\bar{I}$, shown in Fig. 9, is represented by the following simple form of equation:

$$
\bar{I}=1-\exp \left(-\frac{z}{\lambda}\right)
$$

where $z$ denotes the downchannel coordinate and $\lambda$ is the characteristic length. It may be mentioned that $\bar{I}$ is zero (i.e., unmixed state) near the entrance of the micromixer and increases up to unity (i.e., completely mixed state) along the downchannel direction. Eqn. (3) is found to fit the experimental data of Fig. 9 quite well, as represented in Fig. 9. Then, the physical meaning of $\lambda$ is the downchannel length required for the intensity $\bar{I}$ to reach 0.632 from zero. We will consider such a characteristic length, $\lambda$ as a characteristic required mixing length in this study. ${ }^{9,11}$

$\lambda$ for several values of $R e$ were determined by optimal fitting of data in Fig. 9 based on eqn. (3). Plotted in Fig. 10 are the $\lambda$ values so obtained as a function of $R e$. At lower $R e, \lambda$ increases as $R e$ increases. However, $\lambda$ is maintained at around $7 \mathrm{~mm}$ when $R e$ becomes greater than 4 due to the favorable effect of advection on the lamination, i.e., increase of interfacial area, as explained above. It might be mentioned that without such increase of interfacial area, in the case of the T-type micromixer, the required mixing length increases with the decrease of the residence time for diffusion as $R e$ increases. $\lambda$ of the T-type micromixer when $R e \approx 2.28$ was $129.7 \mathrm{~mm} .{ }^{9}$ It should be noted that the required mixing length of the SLM is about 20 times shorter than the T-type micromixer at similar $R e$. These results indirectly show that a high level of efficient mixing takes place in the proposed micromixer, SLM, by combining the two general chaotic mixing mechanisms of splitting/recombination and chaotic advection. 


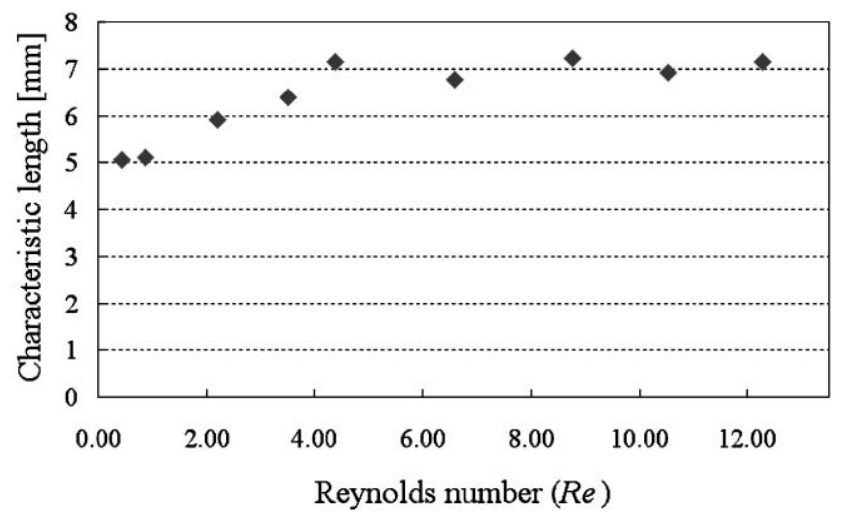

Fig. 10 Characteristic required mixing length as a function of the Reynolds number $(R e)$.

\section{Concluding remarks}

In this paper, we proposed a new passive integrated efficient micromixer, serpentine laminating micromixer (SLM), by combining two general chaotic mixing mechanisms, namely splitting and recombination by the successive arrangement of " $F$ "-shape mixing units in two layers and advection induced by the overall three-dimensional serpentine channel path. Mold inserts for the injection molding process of the SLM were fabricated by SU-8 photolithography and nickel electroplating. The SLM was realized by injection molding of COC and thermal bonding of two injection molded COC substrates. Mixing performance of the SLM was fully characterized numerically and experimentally, from which one can conclude the followings:

(i) With a simple design of successive arranged F-shape mixing units, two efficient chaotic mixing mechanisms were favorably combined. From the cross-sectional simulation results of mixing patterns, it was found that the advection effect from the serpentine channel path design acts favorably towards the vertical lamination of fluid flow as $R e$ increases, which otherwise could have not been realized without a separation wall.

(ii) The first interface which was formed at the T-junction of the SLM flows into the first splitting channel when $R e$ is small, which was observed both numerically and experimentally. Numerical simulation results showed that the first interface tends to flow into the second splitting channel at high $R e$. In the case of intermediate ranges of $R e$, however, the motion of the interface is not so clear at this moment.

(iii) The mixing performance of the SLM was experimentally characterized in a quantitative manner based on colorimetric pixel analysis making use of a $\mathrm{pH}$ indicator, phenolphthalein.

(iv) The mixing experimental results showed that a high level of efficient mixing can be achieved with the SLM for the wide range of $R e$.

It may be mentioned that the detailed combining effect of two chaotic mixing mechanisms of splitting/recombination and chaotic advection is to be investigated further in the future.

It might be also mentioned that a recent parametric study on the mixing performance of the SLM shows that the channel aspect ratio is one of the most important design parameters. By increasing thickness of the recombination region, one can get the better mixing performance. With the same thickness, one can also achieve better performance by changing the geometrical shape of the F-shape mixing unit. Furthermore, it is possible to introduce several splitting channels, instead of the two splitting channels of the present design, as an alternative design. However, one needs special care in this alternative design since the several splitting channel design may cause different flow path lengths of the splitting streams. To prevent this kind of problem, one could adjust the widths of the splitting channels to keep the same pressure drop for all splitting streams.

It may be finally mentioned that due to the simple and mass producible geometry of the efficient micromixer, SLM suggested in this study, the SLM can be easily applied to integrated microfluidic systems, such as micro-total-analysissystems or lab-on-a-chip systems.

\section{Acknowledgements}

The authors would like to thank the Korean Ministry of Science and Technology for financial support via the National Research Laboratory Program (2000-N-NL-01-C-148) and the Korean Ministry of Education \& Human Resources Development supporting BK21 program. The authors appreciate many helpful discussions with Dr Tae Gon Kang at the Department of Mechanical Engineering in POSTECH.

Dong Sung Kim, ${ }^{a b}$ Se Hwan Lee, ${ }^{c}$ Tai Hun Kwon*ab and Chong H. Ahn ${ }^{c}$ ${ }^{a}$ Department of Mechanical Engineering, Pohang University of Science and Technology (POSTECH), San 31 Hyoja-dong Nam-gu, Pohang, Kyungbuk 790-784, Korea

${ }^{b}$ Research Center for Micro Mechanical Systems, Pohang University of Science and Technology (POSTECH), San 31 Hyoja-dong Nam-gu, Pohang, Kyungbuk 790-784, Korea.E-mail: thkwon@postech.edu ${ }^{c}$ MicroSystems and BioMEMS Lab, Department of Electrical \& Computer Engineering and Computer Science, University of Cincinnati, P.O. Box 210030, Cincinnati, OH 45221-0030, USA

\section{References}

1 A. Manz, N. Grabber and H. M. Widmer, Miniaturized total chemical analysis systems: a novel concept for chemical sensing, Sens. Actuators, B, 1990, 1, 244-248.

2 In Micro Total Analysis Systems 2002: Proceedings of the $\mu T A S$ 2002 Symposium held in Nara, ed. Y. Baba, S. Shoji and A. van den Berg, Japan, November 2002, Kluwer Academic Publishers.

3 W. Ehrfeld, V. Hessel and H. Lowe, Microreactors-New Technology for Modern Chemistry, Wiley-VCH, New York, 2000.

4 C.-C. Hong, J.-W. Choi and C. H. Ahn, A novel in-plane passive microfluidic mixer with modified Tesla structures, Lab Chip, 2004, 4, 109-113.

5 T. J. Johnson, D. Ross and L. E. Locascio, Rapid microfluidic mixing, Anal. Chem., 2002, 74, 45-51; T. J. Johnson and L. E. Locascio, Characterization and optimization of slanted well designs for microfluidic mixing under electroosmotic flow, Lab Chip, 2002, 2, 135-140.

6 Y.-K. Lee, J. Deval, P. Tabeling and C.-M. Ho, Chaotic mixing in electrokinetically and pressure driven micro flows, in Proceedings of the IEEE Micro Electro Mechanical Systems, Interlaken, Switzerland, January 2001, pp. 483-486.

7 R. H. Liu, M. A. Stremler, K. V. Sharp, M. G. Olsen, J. G. Santiago, R. J. Adrian, H. Aref and D. J. Beebe, Passive mixing in a three-dimensional serpentine microchannel, J. Microelectromech. Syst., 2000, 9, 190-197.

8 A. D. Stroock, S. K. W. Dertinger, A. Ajdari, I. Mezic, H. A. Stone and G. M. Whitesides, Chaotic mixer for microchannels, Science, 2002, 295, 647-651. 
9 D. S. Kim, S. W. Lee, T. H. Kwon and S. S. Lee, A barrier embedded chaotic micromixer, J. Micromech. Microeng., 2004, 14, 798-805.

10 A. Bertsch, S. Heimagartner, P. Cousseau and P. Renaud, Static micromixers based on large-scale industrial mixer geometry, $L a b$ Chip, 2001, 1, 56-60.

11 D. S. Kim, I. H. Lee, T. H. Kwon and D.-W. Cho, A barrier embedded Kenics micromixer, J. Micromech. Microeng., 2004, 14, 1294-1301.

12 J. Branebjerg, P. Gravesen and J. P. Krog, Fast mixing by lamination, in Proceedings of the IEEE Micro Electro Mechanical Systems, San Diego, USA, February 1996, pp. 441-446.

13 N. Schwesinger, T. Frank and H. Wurmus, A modular microfluid system with an integrated micromixer, J. Micromech. Microeng., 1996, 6, 99-102.

14 F. Schönfeld, V. Hessel and C. Hofmann, An optimized split-andrecombine micro-mixer with uniform 'chaotic' mixing, Lab Chip, $2004,4,65-69$.

15 J. M. Ottino, The Kinematics of Mixing: Stretching, Chaos, and Transport, Cambridge Univeristy Press, New York, 1989.
16 S. J. Kim and T. H. Kwon, Enhancement of mixing performance of single screw extrusion process via chaotic flows, Adv. Polym. Tech., 1996, 15, 41-69.

17 W. R. Hwang and T. H. Kwon, Dynamical modeling of chaos single-screw extruder and its three-dimensional numerical analysis, Polym. Eng. Sci., 2000, 40, 702-714.

18 L. S. Roach, H. Song and R. F. Ismagilov, Controlling nonspecific protein adsorption in a plug-based microfluidic system by controlling interfacial chemistry using fluorous-phase surfactants, Anal. Chem., 2005, 77, 785-796.

19 S. H. Lee, J. Han, D. S. Kim, T. H. Kwon, C. J. Hwang, Y. M. Heo and C. H. Ahn, A high precision self-assembly technique for multilayer polymer lab-on-a-chip, in Proceedings of Micro Total Analysis Systems 2004 ( $\mu$ TAS 2004), Malmö, Sweden, September 2004, vol. 2, pp. 413-415.

20 T. G. Kang and T. H. Kwon, Colored particle tracking method for mixing analysis of chaotic micromixers, J. Micromech. Microeng., 2004, 14, 891-899.

21 S. Hardt and F. Schönfeld, Laminar mixing in different interdigital micromixers: II. Numerical simulations, AIChE J., 2003, 49, $578-584$. 